\title{
The genome of the Lactobacillus sanfranciscensis temperate phage EV3
}

\author{
Matthias A Ehrmann ${ }^{1 *}$, Angel Angelov ${ }^{2}$, Claudia Picozzi ${ }^{3}$, Roberto Foschino ${ }^{3}$ and Rudi F Vogel ${ }^{1}$
}

\begin{abstract}
Background: Bacteriophages infection modulates microbial consortia and transduction is one of the most important mechanism involved in the bacterial evolution. However, phage contamination brings food fermentations to a halt causing economic setbacks. The number of phage genome sequences of lactic acid bacteria especially of lactobacilli is still limited. We analysed the genome of a temperate phage active on Lactobacillus sanfranciscensis, the predominant strain in type I sourdough fermentations.

Results: Sequencing of the DNA of EV3 phage revealed a genome of 34,834 bp and a G + C content of $36.45 \%$. Of the 43 open reading frames (ORFs) identified, all but eight shared homology with other phages of lactobacilli. A similar genomic organization and mosaic pattern of identities align EV3 with the closely related Lactobacillus vaginalis ATCC 49540 prophage. Four unknown ORFs that had no homologies in the databases or predicted functions were identified. Notably, EV3 encodes a putative dextranase.
\end{abstract}

Conclusions: EV3 is the first L. sanfranciscensis phage that has been completely sequenced so far.

Keywords: Genome, Bacteriophage, EMBL accession number PRJEB61, Lactobacillus sanfranciscensis, Sourdough fermentation

\section{Background}

In many large-scale food fermentations manufactured with lactobacilli, the risk of bacteriophage contamination is a serious threat. Phage infections are detrimental in industrial dairy or acetic acid fermentations [1-3], where the liquid state of the medium allows the rapid dissemination of the viral particles. Despite spreading of the phage within a sourdough is hindered, probably as a consequence of the semifluid physical state of the matrix, phages of lactobacilli have been already isolated from sourdough samples $[4,5]$ and it has been proven that viral infection can be transmitted from one dough to another [6]. Interestingly, phage spreading into sourdough did neither adversely affect acidification and volume increase of the dough nor reduced lactobacilli cell counts [5].

In a previous work phage EV3 was isolated and phenotypically characterized, showing to be active on five different strains of $L$. sanfranciscensis [5]. This viral particle

\footnotetext{
* Correspondence: m.ehrmann@wzw.tum.de

'Lehrstuhl für Technische Mikrobiologie, Technische Universität München, Gregor-Mendel-Str. 4, Freising 85354, Germany

Full list of author information is available at the end of the article
}

was ascribed to the Siphoviridae family with a morphotype B1. Its lytic life cycle at $25^{\circ} \mathrm{C}$ lasted $3 \mathrm{~h}$ with a burst size of about 30 viral particles per infected cell. The genome estimated by digestion with different restriction enzymes was $31.8 \pm 1.5 \mathrm{kbp}$ long, and it was a doublestranded linear DNA molecule with a pac-type system. Phage EV3 behaves as a temperate phage that can either multiply via the lytic cycle or enter a dormant state integrating into the host chromosome as a prophage.

Phages may be the most abundant life forms on Earth with a global population on the order of $10^{31}$ [7]. Significant amount of sequencing data is generated by phage genome projects and by sampling of DNA in the environment. Actually, since phages are the main vectors of gene exchange phenomena, they are considered the most important factors in driving evolution in prokaryotes [8].

To date, validated genome sequences of 16 Lactobacillus bacteriophages (including prophages) are available from the National Center for Biotechnology Information (NCBI) reference sequence database (RefSeq). The availability of those data allows for comparison of viral genomes in order to understand the genetic relationships 
among different phages and the function of putative genes. Whereas knowledge on phages and genomes thereof derived from lactic acid bacteria of the dairy environment is increasing, reports on phages coming from cereal fermentations are still rare.

This is the first report of the genome analysis of a $L$. sanfranciscensis phage.

\section{Results \& discussion}

\section{Genome structure}

EV3 phage belongs to the family of Siphoviridae in the order Caudovirales. It had a genome length of $34.834 \mathrm{bp}$ with an overall $G+C$ content of $36.45 \%$. Forty-three possible ORFs are numbered consecutively starting from ORF EV3_001 encoding the terminase gene. Amino acids length of ORFs ranged from 51 amino acids (EV_28) to 1263 aa (EV_013). Six of the 43 ORFs were preceded by perfect matching Shine-Dalgarno sequences with the consensus sequence (AGGAGG) that is generally conserved in lactobacilli and was chosen as the recognition sequence for ribosome binding site (RBS) prediction [9]. The consensus sequence is complementary to the $3^{\prime}$ end of the $16 \mathrm{~S}$ rRNA gene of L. sanfranciscensis (5'-CACCTCCTTTCT-3'). Twenty ORFs showed a 1-mismatch RBS and 16 ORFs show less or no sequence similarity. As concern the start codon, ATG predominates (93\%). Only ORF EV_15 and the two ORFs EV_34 and EV_40 apparently initiated translation with the TTG start codon and the GTG start codon, respectively (Table 1). A putative function based on similarity level to protein with known functions was assigned to 39 ORFs (Table 1). Highest sequences similarities are with phages infecting lactic acid bacteria. In particular, 13 sequences in the late gene cluster had a similarity with the ones found in Lactobacillus vaginalis ATCC 49540 phage whereas six showed correspondence with the ones of Lactobacillus fructivorans KCTC 3543 phage. Forty ORFs were oriented in the same direction while three (orf EV3_023, EV3_0 24 and EV3_025) belonging to the lysogeny module were located on the opposite strand. The genome was organized in five functional clusters: DNA packaging, morphogenesis, lysis, lysogeny and DNA replication (Figure 1). Between morphogenesis and lysis clusters there was a peculiar ORF coding for a dextranase.

\section{EV3 DNA packaging}

The predicted protein products of ORF EV3_01 and EV3_02 were similar to the putative small and large terminase subunits from L. vaginalis ATCC 49540 phage. In tailed phages, terminases consist of a large subunit containing the ATPase activity that controls DNA translocation together with an endonuclease activity that cuts concatemeric DNA into genome lengths, and a small subunit responsible for specific DNA binding. Therefore, these two EV3 proteins were probably involved in DNA packaging. In a previous work [5] it was already highlighted that EV3 had no cos site and therefore it is likely to pack its DNA through a pac system. The protein encoded by ORF EV3_035 had a high similarity with the putative DNA binding protein of $L$. hilgardii ATCC 8290. Its position was quite close to terminases genes suggesting that the putative gene product of ORF EV3_035 could also be involved in DNA packaging.

\section{DNA morphogenesis}

ORF EV3_003 and EV3_004 constituted the putative head module, since they were similar to portal protein and capsid protein of L. vaginalis ATCC 49540 phage, respectively. The portal complex forms a channel through which the viral DNA is packaged into the capsid, and exits during infection. The portal protein is thought to rotate during DNA packaging. It also forms the junction between the phage head (capsid) and the tail proteins. Putative gene products encoded by ORF EV3_007 and EV3_008 were likely to connect head and tail structures. The overlapping of the two genes suggest a translational coupling. The putative tail module was positioned downstream from the predicted head-tailjoining genes, and it was composed by ORF EV3_009, EV3_010. EV3_013 encoded product was similar to various tail component and tape measure proteins (TMP) from phages of $L$. vaginalis and L. fermentum. TMP generally works as template for measuring length during tail assembly, thus, it is reasonable to ascribe this function to the protein.

\section{Lysis module}

The predicted protein product from ORF EV3_021 had a $44 \%$ overall identity with the holin of L. plantarum WCFS1 phage P1. Holins are a diverse family of proteins that cause bacterial membrane lysis during late-protein synthesis. ORF EV3_022 encodes a putative endolysin that is quite similar (54\% identity) to the endolysin of $L$. vaginalis ATCC 49540. The $C$ terminus of this ORF contains two Lysine Motif domains that are likely to be implicated in bacterial cell wall degradation, while the $\mathrm{N}$ terminus encloses a Cpl-1 lysin (also known as Cpl-9 lysozyme/muramidase) that is a bacterial cell wall endolysin. A signal peptide with a predicted cleavage site (probability of 0.750 ) between position 26 and 27 of the amino acid sequence was identified. An analogous signal peptide was already reported for other phages of lactic acid bacteria and was demonstrated to be active $[10,11]$.

\section{Integrase module and attachment site}

ORF EV3_023 has an amino acid sequence comparable to phage integrase of $L$. salivarius ACS-116-V-Col5a 
Table 1 Open reading frames and genetic features of $L$. sanfranciscensis EV3 phage

\begin{tabular}{|c|c|c|c|c|c|c|}
\hline Locus tag & ${\text { Putative } \text { RBS }^{*} \text { and start codon }}^{\neq}$ & nt & $\begin{array}{l}\text { orf length } \\
\text { (aa) }\end{array}$ & Best hit & $\begin{array}{l}\text { Best hit EMBL } \\
\text { protein name }\end{array}$ & Score \\
\hline EV3_001 & ttacgaaaggagaaattgtatg & $136-675$ & 179 & Phage terminase L. vaginalis, small subunit ATCC 49540 & EEJ41449 & $1.0 \mathrm{E}-88$ \\
\hline EV3_002 & gacagtattgctaatatgctaaatg & $650-2590$ & 646 & Phage terminase L. vaginalis, large subunit, ATCC 49540 & EEJ41450.1 & 0.0 \\
\hline EV3_003 & tgcgataggaggtgattagcaatg & $2786-3964$ & 398 & Phage portal protein L. vaginalis ATCC 49540 & EEJ41452.1 & 0.0 \\
\hline EV3_004 & taaaggaggtgataatgtaaatg & $3930-5918$ & 662 & phage capsid protein L. vaginalis ATCC 49540 & EEJ41453.1 & 0.0 \\
\hline EV3_005 & caaaataaaggagtgataaaatg & $5950-6489$ & 179 & major tail protein Staph. pseudintermedius HKU10-03 & ADV05789.1 & $1.0 \mathrm{E}-13$ \\
\hline EV3_006 & actaagggagatgagtagcaatg & $6509-6814$ & 101 & Putative uncharacterized protein L. vaginalis ATCC 49540 & EEJ41454.1 & $6.0 \mathrm{E}-45$ \\
\hline EV3_007 & aacaagggtggtgacagccaatg & $6808-7176$ & 122 & Head-tail joining protein L. vaginalis ATCC 49540 & EEJ41455.1 & $5.0 \mathrm{E}-155$ \\
\hline EV3_008 & tgtaaaggagctgagtggcaatg & $7169-7606$ & 145 & Head-tail joining protein L. vaginalis ATCC 49540 & EEJ41456.1 & 2.0E-91 \\
\hline EV3_009 & aaaggatgttaaatcatgaaaatg & 7603-7992 & 129 & Phage tail protein L. vaginalis ATCC 49540 & EEJ41424.2 & 7.0E-66 \\
\hline EV3_010 & ttagaaaagaggaaataatttatg & $7996-8763$ & 255 & Phage major tail protein L. vaginalis ATCC 49540 & EEJ41425.1 & $1.0 \mathrm{E}-123$ \\
\hline EV3_011 & cggccattaaggagataagtaatg & $8873-9283$ & 136 & Putative uncharacterized protein L. vaginalis ATCC 49540 & EEJ41426.1 & $9.0 \mathrm{E}-64$ \\
\hline EV3_012 & gtcgtaagccaaacgtggctctgatg & $9343-9513$ & 56 & Putative uncharacterized protein L. vaginalis ATCC 49540 & EEJ41427.1 & $7.0 \mathrm{E}-22$ \\
\hline EV3_013 & gaaggaaggaggtaactaaatg & $9514-13305$ & 1263 & Phage minor tail protein L. vaginalis ATCC 49540 & EEJ41428.1 & 0.0 \\
\hline EV3_014 & aacttaatggaggtcttgcataatg & $13305-14141$ & 278 & Putative uncharacterized protein L. salivarius (strain CECT 5713) & ADJ78578.1 & $3.0 \mathrm{E}-48$ \\
\hline EV3_015 & aatttagggaggtgttaatttg & 14161-15396 & 411 & Glycosylhydrolase L. plantarum & CCC78574.1 & $4.0 \mathrm{E}-17$ \\
\hline EV3_016 & aggaacaagggtgattatttaatg & $15396-17177$ & 593 & Put. Minor structural protein Leu. kimchii IMSNU 11154 & ADG39890.1 & $3.0 \mathrm{E}-62$ \\
\hline EV3_017 & tttgtctaggaaggagaaaaaatg & $17190-17726$ & 54 & Hypothetical protein & No hit & - \\
\hline EV3_018 & aaaagttgggagtgattaaaaatg & $17729-20134$ & 801 & Dextranase L. fermentum phage phiPYB5 & ADA798961.1 & $1.0 \mathrm{E}-137$ \\
\hline EV3_019 & atgaaataaaggagaaaataaatg & $20212-20721$ & 170 & hypothetical protein & No hit & - \\
\hline EV3_020 & tggagaaaggaggtgatgaaatg & $20790-21083$ & 97 & Predicted protein P. acidilactici & EFA25777.1 & $2.0 \mathrm{E}-4$ \\
\hline EV3_021 & aaagaaacgagtgaacaatatg & 21067-21309 & 80 & L. plantarum WCFS1 phage P1 holin, Ip0683 & F9ULS1 & $6.0 \mathrm{E}-68$ \\
\hline EV3_022 & taccattaatcatcgttgctgaaatg & $21383-22429$ & 348 & putative endolysin L. vaginalis ATCC 49540 & EEJ39754.1 & $9.0 \mathrm{E}-70$ \\
\hline EV3_023 & aaagcagaaagcgaattaatatg & $23961-22876 c$ & 361 & phage integrase L. salivarius ACS-116-V-Col5a & EFK79983.1 & $1.0 \mathrm{E}-112$ \\
\hline EV3_024 & atattctaaggagaatggaaaatg & $24072-24485 c$ & 137 & Putative uncharacterized protein L. pentosus MP-10 & CCB81756.1 & $4.0 \mathrm{E}-14$ \\
\hline EV3_025 & tagaatacgattggatattgatatg & $24478-24795 c$ & 110 & XRE family transcriptional regulator L. pentosus & FR871768 & $4.0 \mathrm{E}-23$ \\
\hline EV3_026 & tatgaaaaggaggaatcaatatg & $25072-25263$ & 63 & Phage antirepressor L. ruminis ATCC 25644 & EFZ34631.1 & $2.0 \mathrm{E}-10$ \\
\hline EV3_027 & agaaaaaagatgattgtcatg & $25378-25638$ & 89 & Phage protein Listeria monocytogenes FSL N1-017 helix-turn-helix protein & EFK40475.1 & $4.0 \mathrm{E}-5$ \\
\hline EV3_028 & tataagggggtgagatagatg & $25639-25794$ & 51 & Hypothetical protein & No hit & - \\
\hline EV3_29 & attcaaggagatgtaataaatg & $25798-26028$ & 77 & Hypothetical protein & No hit & - \\
\hline EV3_030 & aatctaaaaggaagttattcatatg & $26146-26322$ & 59 & hydrolase NUDIX family L. delbrueckii & CAl96847 & 0.73 \\
\hline EV3_031 & aattttagtgggggtagagaaatg & $26336-26821$ & 161 & Putative uncharacterized protein Mahella australiensis DSM 15567 & AEE95754.1 & $6.0 \mathrm{E}-7$ \\
\hline EV3_032 & ccgaaaggaagtgagataaatg & $26852-27319$ & 155 & Putative uncharacterized protein Ip_0862 L. pentosus IG1 & FR874854.1 & $1.0 \mathrm{E}-22$ \\
\hline EV3_033 & actaaattataggagataaatatg & $27380-28120$ & 246 & NTP-binding protein L. paracasei subsp. paracasei ATCC 25302 & EEl67797.1 & $5.0 \mathrm{E}-71$ \\
\hline EV3_034 & agtttggaagtgatgaaaacggtg & 28068-29459 & 463 & Putative helicase Lactobacilus phage A2 & CAB63670.1 & $1.0 \mathrm{E}-130$ \\
\hline EV3_035 & acaaataggagaaaaatattatg & 29479-30042 & 187 & Single stranded binding protein L. hilgardii ATCC 8290 & EEI25831.1 & $4.0 \mathrm{E}-41$ \\
\hline EV3_036 & aatatatgaaagggaaaatttatg & 30115-32442 & 775 & Phage primase, P4 family L. buchneri NRRL B-30929 & AEB73788.1 & 0.0 \\
\hline EV3_037 & taattttaaggaggaacacaaatg & $32745-33152$ & 135 & Hypothetical protein & No hit & - \\
\hline EV3_038 & atgaaagatgtgatgtctgataatg & $33145-33465$ & 106 & VRR-NUC domain Phage protein L. plantarum JDM1 & ACT61379.1 & $2.0 \mathrm{E}-33$ \\
\hline EV3_039 & tgaatatagtaggagcatttaaatg & $33462-33686$ & 75 & Hypothetical protein & No hit & - \\
\hline EV3_040 & aagattgggagaaaataaccotg & $33676-33849$ & 58 & Hypothetical protein & No hit & - \\
\hline EV3_041 & caatgtaaggaagaatgataatg & $33872-34114$ & 80 & Ribonucleoside-diphosphate reductase 2, Ent. faecalis & AAO80328.1 & $3.0 \mathrm{E}-13$ \\
\hline EV3_042 & ataatcgtttcgttgggggttattatg & $34196-34612$ & 138 & Phage transcriptional regulator Lactobacillus phage phig1e & CAA66778.1 & 3.0E-14 \\
\hline EV3_043 & taaacaaaaggagtagttaatatg & 34666-34833 & 56 & Putative transporter protein L. reuteri ATCC 53608 & CCC04545.1 & 3.0E-14 \\
\hline
\end{tabular}

*Putative ribosomal binding sites are printed in italics.

FPutative start codons are printed in bold. 


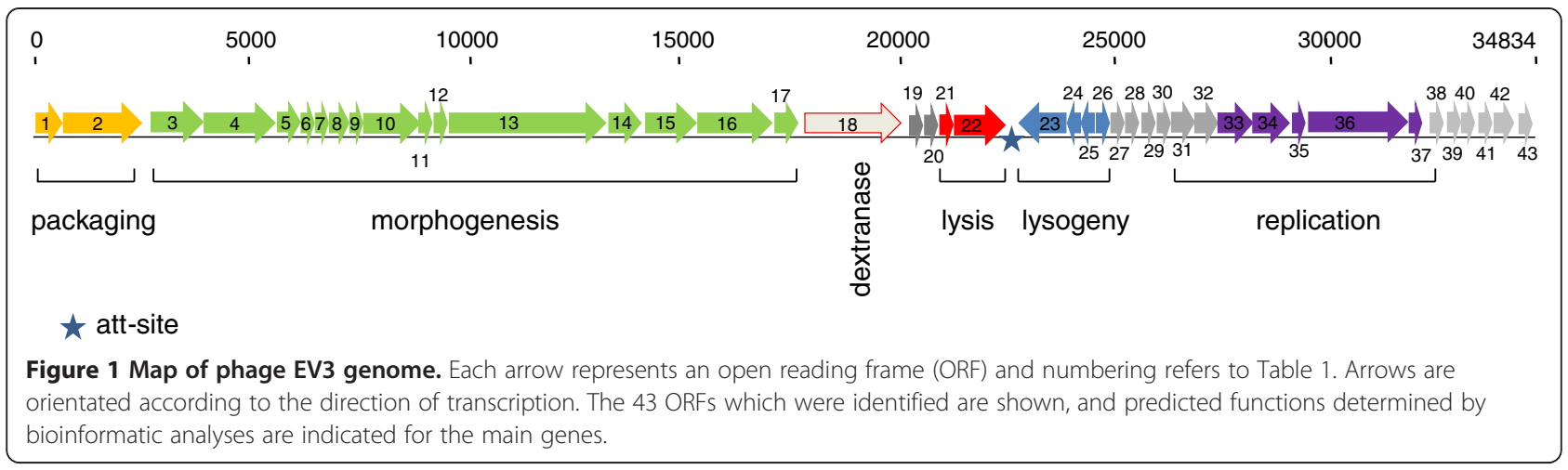

phage. In order to identify the attP site the non coding sequence of 429 bp between the lys (orf EV3_22) and int (orf EV3_23) genes of the phage EV3 was blasted against the whole genome sequence of $L$. sanfranciscensis TMW 1.1304 the only strain whose genome sequence is available [12]. We found only one significant hit of 16 nucleotides matching the $3^{\prime}$ end of a $\mathrm{tRNA}_{\text {Leu }}$ gene. Since the host attachment sites are commonly located near tRNA genes [13], we assumed this sequence as putative attB site in L. sanfranciscensis H2A.

By using primers placed in the bacterial genes flanking the prophage in combination with primers whose binding sites are placed within the int and lys gene, respectively we amplified 658 bp and 370 bp long DNA fragments when DNA of $L$. sanfranciscensis H2A carrying EV3 as a temperate phage was used as template. Sequence analysis revealed that indeed prophage EV3 is located between the putative orf LSA_08690 on the L. sanfranciscensis genome map on one side and a tRNA $\mathrm{Leu}_{\text {Leu }}$ gene on the other (Figure 2). The deduced att $B$ were identical to those to the left and the right, respectively, thus identifying them as the attL and attR sites. Additionally, EV3 is flanked by two 20-bp repeats suggesting a Campbell-like integration of prophage EV3 into the tRNA $A_{\text {Leu }}$ gene that is functionally reconstituted upon prophage integration. Other $L$. sanfranciscensis strains from our collection amplified a 314 bp PCR product when primers were placed in the bacterial genes that bracket the prophage in H2A (data not shown).

Most probably the sequence: 5' GCCGAGAGCGGG 3' found on L. sanfranciscensis genome, is the region recognised by the bacteriophage (att $\mathrm{B}$ site) since an homologous region was found also on EV3 genome (attP site). The att $\mathrm{B}$ region, located between the Lysin and the Integrase genes in lactobacilli containing the phage, corresponds to a gene encoding for a tRNA confirming that some phages integrate their genome directly into genes for the tRNA.

\section{Lysogeny}

The protein encoded by ORF EV3_025 is similar to XRE family transcriptional regulator of $L$. pentosus MP- 10 .
This large family of DNA binding helix-turn helix proteins includes Cro and CI. The product encoded by EV3_026 shows an identity with phage antirepressor of L. vaginalis ATCC 49540 phage. This protein is thought to promote transcription of genes required for phage production.

\section{Phage replication module}

ORF EV3_027 had a DNA binding domain in the Nterminal region with an identity to excisionase protein (Xis protein) and a helix-turn-helix (HTH) DNA binding domain. The predicted proteins from EV3_028 to EV3_032 have an unknown function or they are not characterized. ORF EV3_033 and EV3_034 encode for a phage-DNA binding protein and a helicase, respectively.

\section{Dextranase gene}

The gene is active since it was experimentally shown that clones of H2A strain hosting EV3 phage become dextranase positive [14]. To our knowledge this is the second time that a gene encoding for this enzyme has been found in the phage genome of a lactobacillus [15]. Looking at the position of dextranase gene in the sequences of the viral genome we could speculate that such enzymatic activity can help the viral particle in breaking through dextran producing strains after cell lysis occurs.

Temperate phages are known to carry virulence genes that contribute to the "success" of pathogenic bacteria. There is also a substantial scientific literature explaining this phenomenon by evolutionary arguments [16]. It could well be that temperate phages play this role only for pathogenic bacteria. However, some theoretical reasoning suggests that prophages from non-pathogenic bacteria should encode more general fitness genes that are of selective benefit to the lysogen and/or the host, albeit up to know there is no direct evidence demonstrating prophages encoded fitness factors on of bacterial commensals or food microbes. The present manuscript may be one of the best hints so far for such a fitness factor in the field of LAB, which comprise many 


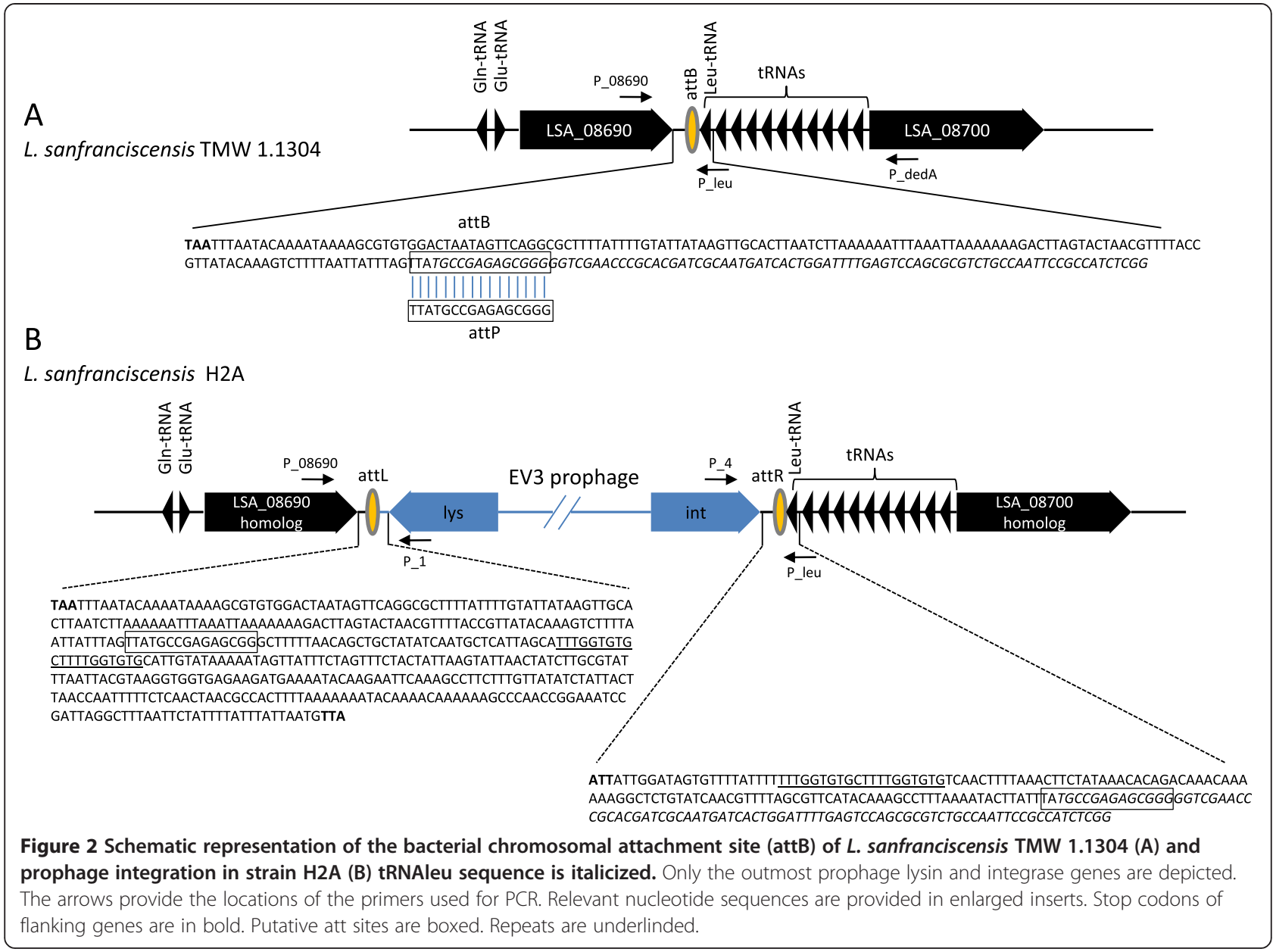

industrially important food bacteria. The demonstration of such a fitness factor could thus have an important impact on theoretical reasoning about the role of phages for the evolution of bacteria in general.

\section{Phyogeny}

The phylogenetic position of EV3 was evaluated using the large subunit of the terminase gene as well as the large subunit portal protein gene. These genes have been previously established as valuable marker for phage phylogeny $[17,18]$. Both marker genes positioned EV3 to a monophyletic group together with phages identified in the genomes of $L$. vaginalis, L. fermentum, L. jensenii, L. rhamnosus and L. casei. These species are neither the closest relatives to $L$. sanfranciscensis nor typically isolated from the sourdough ecosystem. This result may be reflected by the current unavailability of genome sequences of lactobacilli adapted to sourdough fermentations.

\section{Conclusions}

To our knowledge, this study represents the first complete genome sequence and genetic characterization of a $L$. sanfranciscensis phage. Bioinformatic analysis revealed that phage EV3 is a unique temperate phage compared to phages infecting related species of LAB. The endolysin gene was preceeded by a holin gene. The tail morphogenesis module is interspersed with cell lysis genes. The overall amino acid sequences of the phage proteins had little similarity to other sequenced phages. The phage carries a dextranase gene whose function in establishing a stable relationship with their host (lysogen) and influencing its lifestyle and fitness in sourdough fermentations remains to be elucidated. The results of this study may provide new insights that deepen our understanding of phage genetics and phage-host interactions in dynamic ecosystem such as cereal fermentations.

\section{Availability of supporting data}

The phage EV3 genome sequence is deposited at EMBL accession number PRJEB61 http://www.ebi.ac.uk/ena/ data/view/display=html\&PRJEB61.

\section{Methods}

\section{Isolation of phage DNA}

L. sanfranciscensis $\mathrm{H} 2 \mathrm{~A}$ strain was used as host culture for viral multiplication. Phage DNA was isolated from a 
high-titer phage lysate obtained by cesium chloride gradient according to Sambrock et al., [19].

\section{Sequencing strategy}

For full sequencing, purified phage DNA was fragmented by ultrasonification, and ligated into the plasmids pBluescriptKSII and pSmart. Escherichia coli DH5a cells were transformed and colonies were selected by blue/ white selection.

Sequencing was performed on $3 \times 96$ shotgun clones by Sanger sequencing, resulting in a sixfold genome coverage.

Remaining gaps were closed by a Two-Step Gene Walking technique based on randomly primed polymerase chain reaction (PCR) as previously described by Pilhofer et al., [20]. Amplification were performed by use of Kapa2G-Robust Polymerase (Kapa Biosystems, Inc.). It presents a simple workflow, which comprises only two major steps of a Walking-PCR with a single specific outward pointing primer (step 1) and the direct sequencing of its product using a nested specific primer (step 2). Open reading frames (ORFs) were predicted with GeneMark.hmm for Prokaryotes, Version 2.4 [21]. All ORF predictions were verified and modified by blasting ORFs to NCBI nrdb. Additionally, the predicted start codons of all ORFs were inspected manually using the Artemis program [22]. This genome project has been deposited in the European Molecular Biology Laboratory (EMBL)/ Gen- Bank under the accession number PRJEB61. The presence of signal peptides was analysed with SignalP (http://www.cbs.dtu.dk/services/SignalP/).

\section{Determination of the attachment site on the host genome}

In order to identify the attP site we blasted the sequence of 429 bp between the lys and int genes of the phage EV3 against the whole genome sequence of $L$. sanfranciscensis TMW 1.1304 [12].

Primers P_08960 (5' -ATGGAAAAATCGATGTATG) and P_leu (5'-GCCGAGATGGCGGAATTG) placed in the bacterial genes flanking the prophage (orf LSA_08960 and Leu-tRNA), and primers P4 (5'-CGTCGATATTTA TATCATTAG) and P1 (5'-GATACCTTAACCAGAT TAAG) running out of the int and lys genes we amplified 658 bp and 370 bp long DNA fragments, respectively (Figure 2).

\section{Competing interests}

The authors declare that they have no competing interests.

\section{Authors' contributions}

MAE carried out the molecular genetic studies, participated in the genome sequencing and drafted the manuscript. RFV participated in its design and coordination and participated in drafting the manuscript. AA performed the genome annotation. CP participated in the design of the study and drafting the manuscript. RF conceived of the study, and helped to draft the manuscript. All authors read and approved the final manuscript.

\section{Acknowledgements}

We thank Monika Hadek for her technical assistance in laboratory work.

\section{Author details}

'Lehrstuhl für Technische Mikrobiologie, Technische Universität München, Gregor-Mendel-Str. 4, Freising 85354, Germany. ${ }^{2}$ Lehrstuhl für Mikrobiologie, Technische Universität München, Emil-Ramann-Str. 4, Freising 85354, Germany. ${ }^{3}$ Dipartimento di Scienze per gli Alimenti, la Nutrizione e l'Ambiente, Università degli Studi di Milano, DeFENS, via Celoria, Milano 2 20133, Italy.

Received: 2 September 2013 Accepted: 29 November 2013

Published: 5 December 2013

\section{References}

1. Brussow H, Desiere F: Phages of dairy bacteria. Annu Rev Microbiol 2001, 55:283-303

2. Emond E, Moineau S: Bacteriophage Genetics and Molecular Biology. Norfolk UK: Caister Academic Press; 2007.

3. Garneau JE, Moineau S: Bacteriophages of lactic acid bacteria and their impact on milk fermentations. Microb Cell Fact 2011, 10:1-20.

4. Foschino R, Perrone F, Galli A: Characterization of two virulent Lactobacillus fermentum bacteriophages isolated from sour dough. J Appl Bact 1995, 79:677-683.

5. Foschino R, Venturelli E, Picozzi C: Isolation and characterization of a virulent Lactobacillus sanfranciscensis bacteriophage and its impact on microbial population in sourdough. Curr Microbiol 2005, 51:413-418.

6. Foschino R, Galli A, Pagani A, Ottogalli G: Isolation and characterization of bacteriophages active on heterofermentative lactobacilli in sourdoughs. Microbiol Alim Nutr 1996, 14:15-22.

7. Hendrix RH: Bacteriophages: evolution of the majority. Theor Popul Biol 2002, 61:471-480.

8. Weinbauer MG, Agis M, Bonilla-Findji O, Malits A, Winter C: Bacteriophages in the environment. Norfolk, UK: Caister Academic Press; 2007.

9. Pouwels PH, Leer RJ: Genetics of lactobacilli: plasmids and gene expression. Antonie Van Leeuwenhoek 1993, 64:85-107.

10. Durmaz E, Miller MJ, Azcarate-Peril MA, Toon SP, Klaenhammer TR: Genome sequence and characteristics of Lrm1, a prophage from indudtrial Lactobacillus rhamnosus strain M1. Appl Environm Microbiol 2008, 74:4601-4609.

11. Sao-Jose C, Parreira R, Vieira G, Santos MA: The N-terminal region of the Oenococcus oeni bacteriophage fOg44 lysin behaves as a bona fide signal peptide in Escherichia coli and as a cis-inhibitory element, preventing lytic activity on oenococcal cells. J Bacteriol 2000, 182:5823-5831.

12. Vogel R, Pavlovic M, Ehrmann M, Wiezer A, Liesegang H, Offschanka S, Voget S, Angelov A, Böcker G, Liebl W: Genomic analysis reveals Lactobacillus sanfranciscensis as stable element in traditional sourdoughs. Microb Cell Fact 2011, 10(Suppl 1):S6.

13. Williams KP: Integration sites for genetic elements in prokaryotic tRNA and tmRNA genes: sublocation preference of integrase subfamilies. Nucleic Acid Res 2002, 30:866-875.

14. Picozzi C, Meißner D, Foschino R, Vogel RF: Dextranase gene transferred by a Lactobacillus sanfranciscensis phage. In Book of Abstracts of $\mathrm{VI}$ Symposium on Sourdough 14-17 October 2009. Edited by Vogel RF. Freising: Vogel RF; 2009:22.

15. Zhang X, Wang S, Guo T, Kong J: Genome analysis of Lactobacillus fermentum temperate bacteriophage $\varphi$ PYB5. Int J Food Microbiol 2011, 144:400-405.

16. Fortier L-C, Sekulovic O: Importance of prophages to evolution and virulence of bacterial pathogens. Virulence 2013, 4:354-365.

17. Sullivan MB, Coleman ML, Quinlivan V, Rosenkrantz JE, Defrancesco AS: Portal protein diversity and phage ecology. Environ Microbiol 2008, 10:2810-2823

18. Rao VB, Feiss M: The bacteriophage DNA packaging motor. Ann Rev Genet 2008, 42:647-681.

19. Sambrook J, Fitsch EF, Maniatis T: Molecular Cloning: A Laboratory Manual. Cold Spring Harbor: Cold Spring Harbor Press; 1989.

20. Pilhofer M, Bauer AP, Schrallhammer M, Richter L, Ludwig W, Schleifer KH, Petroni G: Characterization of bacterial operons consisting of two tubulins and a kinesin-like gene by the novel two-step gene walking method. Nucleic Acids Res 2007, 35:e135. 
21. Borodovsky M, Mills R, Besemer J, Lomsadze A: Prokaryotic gene prediction using GeneMark and GeneMark.hmm. Curr Protoc Bioinformatics 2003, Chapter 4(Unit4):5.

22. Rutherford K, Parkhill J, Crook J, Horsnell T, Rice P, Rajandream MA, Barrell B: Artemis: sequence visualization and annotation. Bioinformatics 2000, 16:944-945.

doi:10.1186/1756-0500-6-514

Cite this article as: Ehrmann et al:: The genome of the Lactobacillus sanfranciscensis temperate phage EV3. BMC Research Notes 2013 6:514.

\section{Submit your next manuscript to BioMed Central} and take full advantage of:

- Convenient online submission

- Thorough peer review

- No space constraints or color figure charges

- Immediate publication on acceptance

- Inclusion in PubMed, CAS, Scopus and Google Scholar

- Research which is freely available for redistribution 